$$
\text { Conf-950472--10 }
$$

UCRL-JC-120866

PREPRINT

\title{
UV/Visible Camera for the Clementine Mission
}
J. F. Kordas
I. T. Lewis
R. E. Priest
W. T. White III
D. P. Nielsen

\author{
H.-S. Park \\ B. A. Wilson \\ M. J. Shannon \\ A. G. Ledebuhr \\ L. D. Pleasance
}

This paper was prepared for submittal to the SPIE Proceedings

Orlando, FL

April 17-21, 1995

April 1995

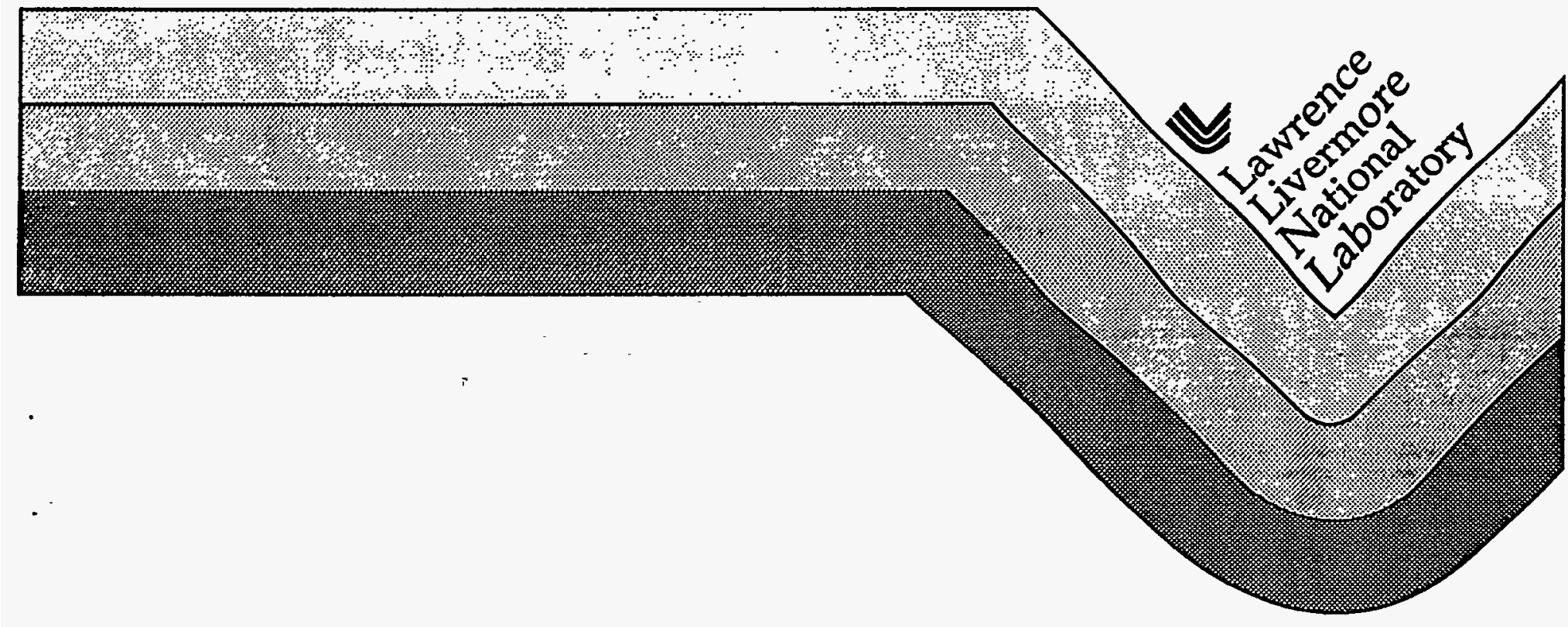




\section{DISCLAIMER}

This report was prepared as an account of work sponsored by an agency of the United States Government. Neither the United States Government nor any agency thereof, nor any of their employees, make any warranty, express or implied, or assumes any legal liability or responsibility for the accuracy, completeness, or usefulness of any information, apparatus, product, or process disclosed, or represents that its use would not infringe privately owned rights. Reference herein to any specific commercial product, process, or service by trade name, trademark, manufacturer, or otherwise does not necessarily constitute or imply its endorsement, recommendation, or favoring by the United States Government or any agency thereof. The views and opinions of authors expressed herein do not necessarily state or reflect those of the United States Government or any agency thereof. 


\section{DISCLAIMER}

Portions of this document may be illegible in electronic image products. Images are produced from the best available original document. 
UV/Visible Camera for the Clementine Mission

\author{
Joseph F. Kordas, Isabella T. Lewis, Robert E. Priest, \\ W. Travis White II, Darron P. Nielsen, Hye-Sook Park, Bruce A. Wilson, \\ Michael J. Shannon, Arno G. Ledebuhr and Lyn D. Pleasance \\ Lawrence Livermore National Laboratory \\ Livermore, California, 94550
}

\begin{abstract}
This article describes the Clementine UV/Visible (UV/Vis) multispectral camera, discusses design goals and preliminary estimates of on-orbit performance, and summarizes lessons learned in building and using the sensor. While the primary objective of the Clementine Program was to qualify a suite of 6 light-weight, low power imagers for future Department of Defense flights, the mission also has provided the first systematic mapping of the complete lunar surface in the visible and near-infrared spectral regions. The $410 \mathrm{~g}, 4.65 \mathrm{~W}$ UV/Vis camera uses a $384 \times 288$ frame-transfer silicon CCD FPA and operates at 6 user-selectable wavelength bands between 0.4 and $1.1 \mu \mathrm{m}$. It has yielded lunar imagery and mineralogy data with up to $120 \mathrm{~m}$ spatial resolution (band dependent) at $400 \mathrm{~km}$ periselene along a $39 \mathrm{~km}$ cross-track swath.
\end{abstract}

Keywords: Clementine UV/Visible Camera, Lunar Imagery, Imaging Sensors, Lunar Mineral Typing.

\title{
INTRODUCTION
}

The Clementine UV/Visible (UV/Vis) sensor is a modification of earlier UV/Vis cameras developed under the Ballistic Missile Defense Program (formerly SDI) for Brilliant Pebbles. It uses a 384 × 288 pixel silicon frame transfer CCD FPA and incorporates 3 selectable gains and 32 selectable offset states in the electronics packaging. A 6-position filter wheel holds six transmissive optical filters, whose pass bands have been chosen to enhance spectral discrimination among key lunar minerals and so provide the most accurate mineral-mapping possible. There is also a broad-band (clear) filter chosen to maximize detection range for a rendezvous with the asteroid Geographos. The f/1.96 catadioptric lens was designed for compactness and for good signal-to-noise ratio both for lunar mapping and for detecting Geographos at large distances. To survive the planned 7-month long mission, this camera incorporates radiation-resistant materials and adheres to applicable space design practices.

In addition to generating a data set for lunar mapping, the UV/Vis camera has provided data for sensor lifetime performance on a space platform and provided valuable design lessons for future projects. The remainder of the paper summarizes the Clementine mission and goals, describes the camera construction, operation and testing, and presents some operational results and conclusions.

\section{CLEMENTINE MISSION}

\section{Mission Goals}

The primary objective of the Clementine Program was to demonstrate certain aspects of lightsat technology of interest to the Ballistic Missile Defense Organization (BMDO), including the space qualification of a suite of 6 light-weight, low power imagers [Refs 1, 2, 3 and 4] for future Department of Defense flights. A secondary objective was to produce data of interest to the scientific community. Science missions centered on mineralogical mapping with $100 \%$ coverage of the lunar surface (which was successfully completed), and spectral studies of the near-Earth asteroid Geographos (which was not completed). Studies of radiation environment effects, camera noise under spacecraft platform control, and all lifetime issues were also performed.

\section{Mission Synopsis}

The Clementine spacecraft launched on schedule on January 25, 1994 from Vandenberg Air Force Base (CA). After 25 days in lunar transit, which included a week in low earth orbit and the remainder in phasing loops, the spacecraft was inserted into an elliptical polar lunar orbit where it successfully spent 71 days performing a systematic mapping of the Moon. The 
spacecraft left the Moon on May 4, 1994 and was in the middle of a pair of orbital loops around the Earth preparatory to obtaining a gravity-assist boost from the Moon towards Geographos when a software failure caused complete loss of attitude control system propellant and put the spacecraft in an 81-rpm spin. The spacecraft could not be despun to a low enough rate to permit further acquisition of resolvable images, nor could the spacecraft be pointed to a specified direction. As a result there was no possibility of completing the Geographos phase of the mission. Refs $[5,6]$ provide good overviews and insight into utility of the Clementine data that have been analyzed.

\section{DESCRIPTION}

Fig. 1 depicts the UV/Vis camera. The unit consists of an optical system (lens assembly and light baffle), a camera module, and a filter wheel module, each of which is discussed below. Table 1 summarizes the camera's technical specifications.

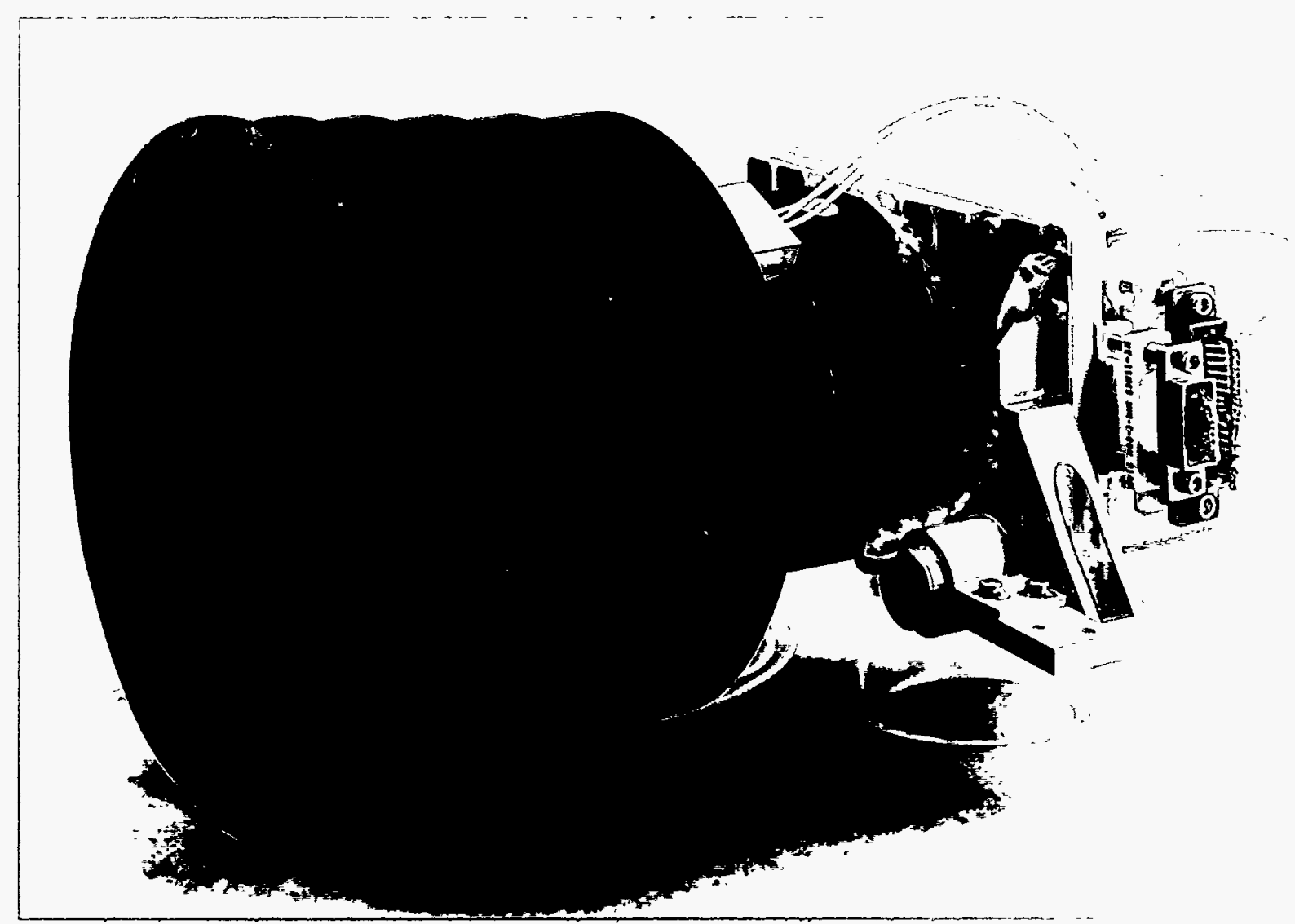

Figure 1. Clementine UV/Visible camera. 
Table 1. Clementine UV/Visible Camera Specifications.

\begin{tabular}{|c|c|}
\hline Attribute & Characteristic \\
\hline $\begin{array}{l}\text { Focal Plane Arrays } \\
\text { Type } \\
\text { Pixel Format } \\
\text { Array Size } \\
\text { Pixel Size } \\
\text { CCD Operating Temperature }\end{array}$ & $\begin{array}{l}\text { Thomson TH7863-CRH-UV-B/T } \\
\text { Si CCD - } 0.2 \text { to } 1.1 \text { microns } \\
\text { Frame Transfer Device } \\
384 \times 288 \\
8.83 \times 13.25 \mathrm{~mm} \\
23 \times 23 \text { microns } \\
10^{\circ} \mathrm{C} \text { Maximum (Clementine spacecraft } \\
\text { specific) }\end{array}$ \\
\hline $\begin{array}{l}\text { Optics } \\
\text { Type } \\
\text { Aperture } \\
\text { Focal Length } \\
\text { Speed }\end{array}$ & $\begin{array}{l}\text { Catadioptric } \\
46 \mathrm{~mm} \text { outer diameter. } \\
90 \mathrm{~mm} \\
\text { F/1.96 }\end{array}$ \\
\hline $\begin{array}{l}\text { Imaging } \\
\text { Spectral Range } \\
\text { Array FOV } \\
\text { IFOV } \\
\text { Point Spread } \\
\text { Clementine Filters }\end{array}$ & $\begin{array}{l}0.3 \text { to } 1.1 \text { microns } \\
4.2^{\circ} \times 5.6^{\circ} \\
255 \mu \mathrm{rad} \\
1.1 \text { to } 1.5 \text { pixels } \\
415 \mathrm{~nm}( \pm 20 \mathrm{~nm} \text { bw }), 750( \pm 5), 900 \\
( \pm 10), 950( \pm 15), 1000( \pm 15), 400 \text { to } \\
950 \text { broadband }\end{array}$ \\
\hline $\begin{array}{l}\text { Electronics } \\
\text { A/D Resolution } \\
\text { Frame Rate } \\
\text { Readout Time } \\
\text { Digitization (äpproximate) } \\
\text { Readout Noise } \\
\text { Integration Time } \\
\text { Offset Control } \\
\text { Average Power }\end{array}$ & $\begin{array}{l}8 \text { bits } \\
<10 \mathrm{~Hz} \\
27.4 \mathrm{msec} \\
150,350,1000 \mathrm{e}^{-/ \text {count }} \\
60-75 \mathrm{e}^{-\mathrm{RMS}} \\
0.2-773 \mathrm{~ms} \\
248 \text { gray level offset; } 5 \text {-bit control } \\
\text { word; LSB = } 8 \text { gray levels } \\
4.5 \text { Watts }\end{array}$ \\
\hline $\begin{array}{l}\text { Filter Wheel System } \\
\text { Type } \\
\text { Step and settle time } \\
\text { Position repeatability } \\
\text { Power }\end{array}$ & $\begin{array}{l}6 \text { position, } 90^{\circ} \text { stepper motor driven, } \\
\text { Hall effect position sensors } \\
\leqslant 250 \mathrm{~ms} \\
\leqslant 10 \mathrm{mr} \\
0.15 \mathrm{~W} \text { quiescent, } 11.0 \mathrm{~W} \text { stepping }\end{array}$ \\
\hline $\begin{array}{l}\text { Physical } \\
\text { Envelope } \\
\text { Mass }\end{array}$ & $\begin{array}{l}15.5 \mathrm{~cm} \times 11.7 \mathrm{~cm} \times 10.4 \mathrm{~cm} \\
410 \mathrm{~g}\end{array}$ \\
\hline
\end{tabular}

\section{Camera Construction}

As previously identified, this camera has three modules: camera module, filter wheel module and optical system. These modules are seen in the "exploded" assembly photograph of Fig. 3. The camera was designed to survive a $19.8 \mathrm{~g} \mathrm{rms,} 60$ second duration launch aboard a Titan II G vehicle, and operate in space for the seven month duration Clementine mission. 


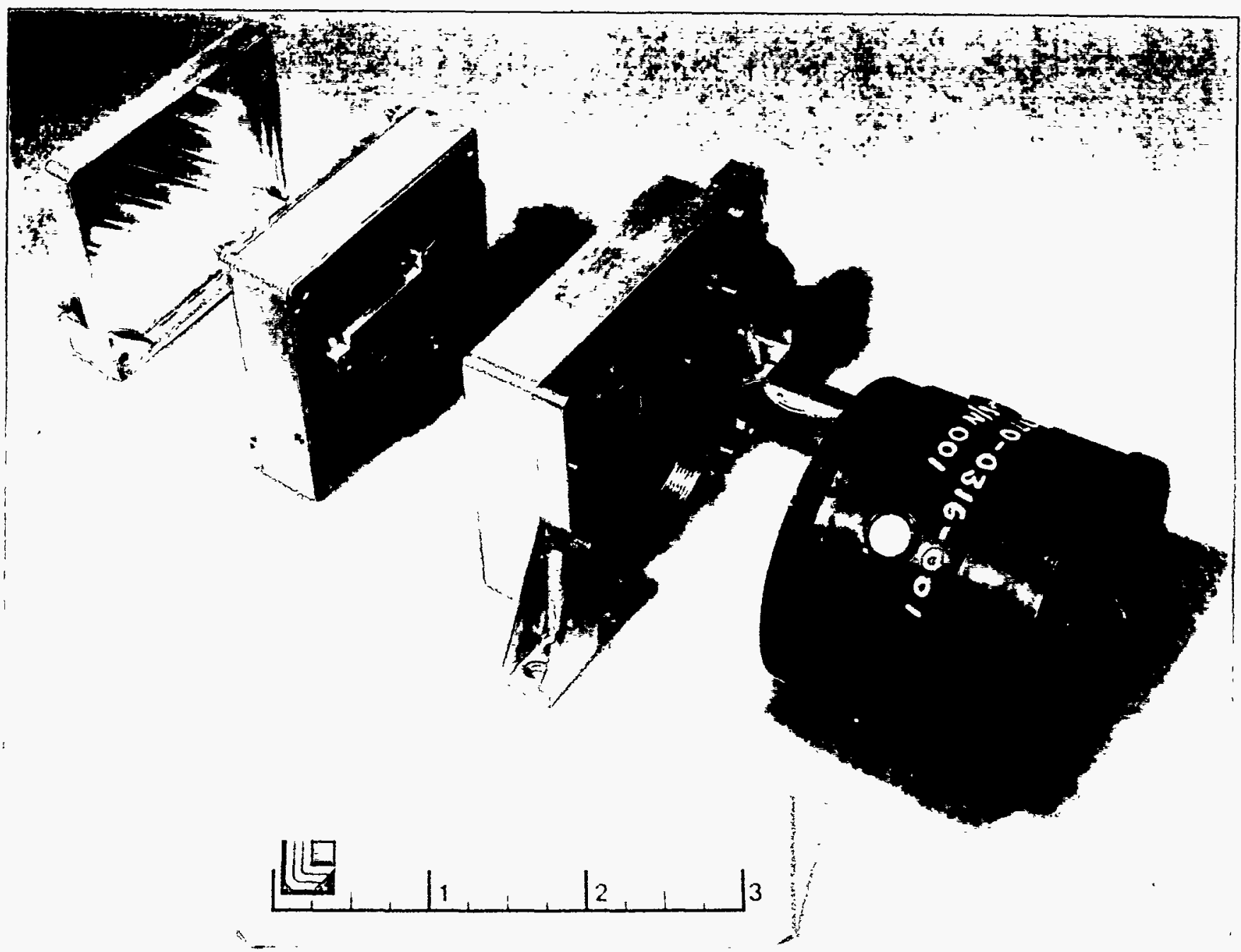

Figure 2. The first prototype Clementine UV/Vis camera shown in modules. From left to right: the end cover which is part of the camera module; the camera module with the CCD showing; the filter wheel module with a filter visible; and the lens (baffle not shown).

\section{Optical System}

Fig. 3 shows the optical system. The UV/Visible lens has a $5.6^{\circ} \times 4.2^{\circ}$ field of view. The lens design is a minor modification of an earlier version of flight hardware. The requirements for the field of view and the broad spectral range required a slight change in the catadioptric design from previous generation lenses. All refractive elements in the design are fused silica, which allows transmission and good aberration correction for all spectral inputs detectable by the UV-enhanced silicon CCD from 0.2 to $1.1 \mu \mathrm{m}$. The science goals and the selection of specific spectral bands are limited by this spectral range.

Ruggedization of the lens assembly has been achieved by using RTV adhesive to pot the individual elements into an aluminum barrel and choosing the RTV thickness so as to compensate for the difference between the thermal contraction of the housing and lens materials. RTV serves as the sole fastening means between the lens elements and the cell, and has successfully survived pre-launch tests, launch, and stage separation. The primary mirror is made of aluminum for passive athermalization of the optical assembly.

The filters used in the camera provide $10^{-4}$ out-of-band rejection, which limits out-of-band light to less than $1 \%$ of the inband signal over typical scene spectra. 


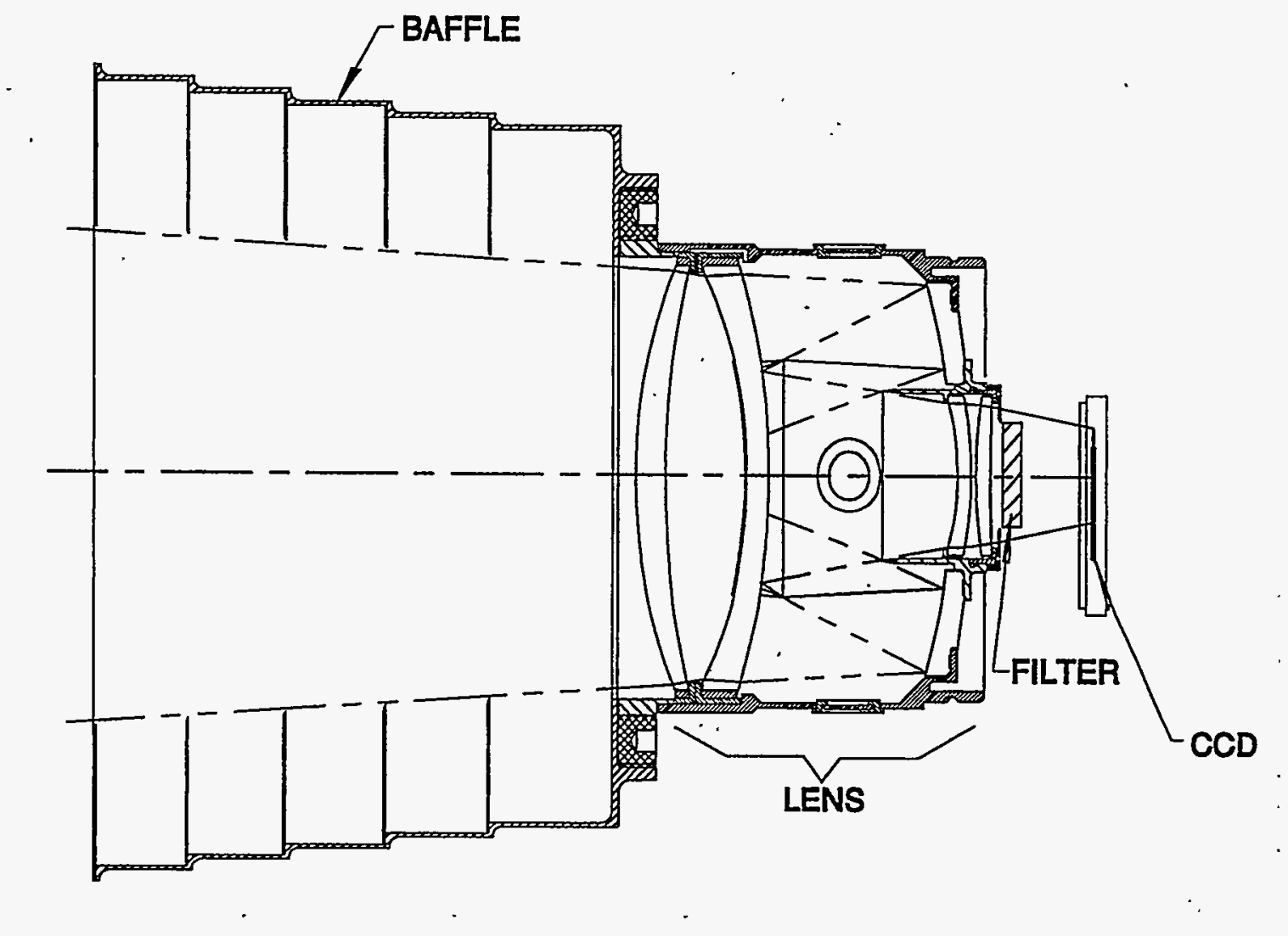

Figure 3. Clementine UV/Visible optical system.

\section{Stray Light Suppression}

Stray light can be important in two situations: first when the camera points near the Sun and second when the camera points at a bright extended scene in which the total reflected flux integrated over all angles is comparable to the solar flux. The first of these scenarios would have occurred during the rendezvous with Geographos, when the Sun was expected to come within about $30^{\circ}$ of the line of sight. The second did occur during lunar mapping, when the UV/Vis viewed a very bright extended scene: namely, a lunar landscape with the Sun near maximum elevation (behind the satellite) as seen from the imaged area of Moon.

The UV/Vis optics have a set of internal and external baffles to suppress veiling glare from sources out of the field of view of the imager. The internal baffling consists of a secondary mirror "collar" and a cone extending from the hole in the center of the primary. Surface finish for the internal baffles is a flat black anodization of the aluminum substrate, chosen because of the handling requirements for these pieces. The external baffle extends 3 inches past the vertex of the leading lens element, and is finished with a MIL-F-495 Copper-black process, which yields reflectivity as low as industry standards Ball black and Martin black processes.

The baffle is shown as part of Fig. 1 and Fig. 3. All material is copper oxide black Al 7075. The baffle is fabricated by machining the cylinder with support steps for the vanes, and machining the $0.010^{\prime \prime}$ thick vanes. With the outside diameter of the vanes undersized to the baffle inside diameter by 0.020 inches, each vane is successively bonded to the cylinder at it's outside diamter with a low outgassing adhesive. The aluminum is electrolis deposited with a .0001 " to .0002 " nickel protectant then electro-plated with $.0003^{\prime \prime}$ copper using a geometry-specific anode. This is then put in a boiling Ebonol soultion at $214^{\circ} \mathrm{F}$ for an experimentally derived period of time (typically tens of minutes). A deionized water rinse, and air drying completes the process. At final camera assembly a single layer of silver teflon tape is applied to the outer surface of the outer vane for in-flight radiative thermal control. 


\section{Camera Module}

The camera module (Ref. [1]) is the chassis and core electronic unit of this camera. It is also the basic building block of the Clementine star tracker and Clementine HiRes cameras. It contains the CCD (Thomson TH7863) and controlling circuitry on a flexible printed wiring assembly (PWA) which communicates via a Synchronous Addressable Serial Interface (SASI) bus protocol to the sensor processor.

The electronics are designed around the CCD, a CCD controller (Thomson TH7990), and an Actel FPGA. The camera sends and receives commands from the Clementine Sensor Imaging Processor (SIP) via a differential line driver/receiver. A SASI receiver interprets all commands. The Actel FPGA then processes all commands, generates video timing, and controls operation of the TH7990, which in turn controls the CCD. The FPGA also controls the analog circuits for Gain, Offset and Double Correlated Sampling (DCS). After decoding each SASI command, the camera returns a status word, and after capturing an image, the camera returns digitized video to the SIP. This process is represented functionally in Fig. 4.

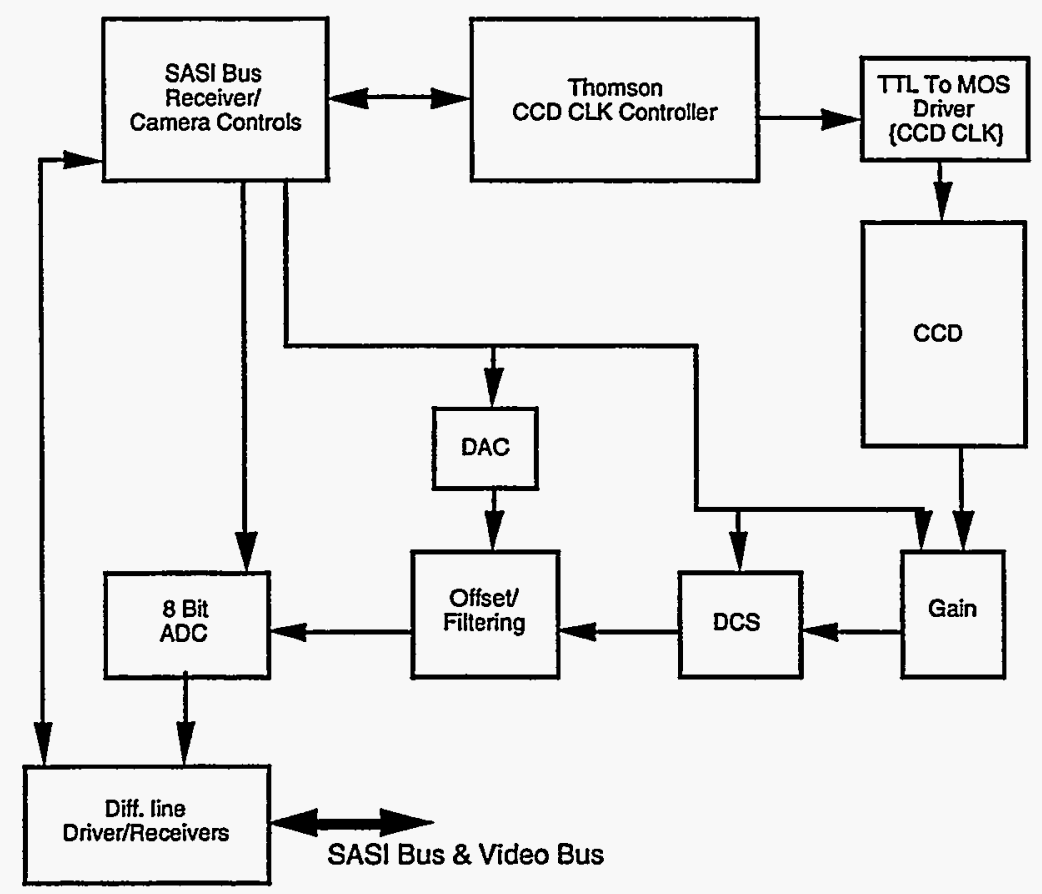

Figure 4. Actel camera electronics functional block diagram.

The camera has two modes of integration control, called "precision integration control" and "SASI control." Control bit 3 of parameter ID 0 selects the mode: bit 3 is HI for precision control, and it is LOW for SASI control. If precision integration control is selected, 13 bits of integration data must be loaded into the command data registers at parameter ID 2 and 3 with bit 4 of the command data register at ID 3 being the most significant bit. Integration time in this mode equals $(n+1) * 94.4$ $\mu$ seconds. The minimum acceptable value for the integer $n$ is 1 , resulting in a minimum integration time for the UV/Vis camera of $188.8 \mu$ seconds. In the SASI control mode, integration is initiated by bringing the bit 0 of the parameter ID 1 to a LOW state, for at least $100 \mu$ seconds. Readout is initiated by bringing the same bit $\mathrm{HI}$. In the precision control mode, integration is initiated by a HI to LOW transition of the same control bit. Readout occurs automatically after the preset integration time. The control bit must be returned $\mathrm{HI}$ before initiating the next integration cycle and left $\mathrm{HI}$ for at least 100 $\mu$ seconds.

In the analog domain, the output of the CCD is buffered through a low-gain emitter-follower amplifier before global gain is applied. The camera has three gain settings (Table 1). Camera gain is selected by setting one of the gain control bits $\mathrm{HI}$ which switches a gain resistor. After applying global gain to the image signal, the camera performs DCS. Gain is applied prior to the DCS to avoid adding noise that would then be amplified. Offset is applied to the signal after DCS. Camera offset is selected by setting a 5-bit control word, which is sent to a digital-to-analog converter (DAC). One least significant bit equals an offset of about 8 gray levels. The maximum offset equals 248 gray levels. After offset the signal is converted to a digital value by the flash analog-to-digital converter. 


\section{Six-Position Filter Wheel System}

Six spectral bands are selectable from a filter wheel which is controlled through the SASI bus. Six filter positions are available using a space-rated $90^{\circ}$ No. 5 stepper motor geared at 6:1. A series of Hall Effect sensors and magnets are used for position determination. A small electronics card for command and operation makes up the balance of the filter positioning system. Filter wheel control is limited to moving forward or backward one filter position at a time and reporting back the present filter position. Both the control logic and interface are implemented in a single Actel FPGA. The filter position control board is a rigid flex printed circuit board with dual thermal planes. This system is modular and functionally separate from the imaging camera. This module with minor configuration modifications is used on the Clementine HiRes and NIR cameras, Refs $[2,4]$.

The basic filter wheel module, fabricated primarily from aluminum, is shown in Fig. 5. The filter wheel subassembly consists of a 2.125" pitch wheel with 204 teeth fabricated to AGMA class 10, the 6 filters, 9 magnets, and two ABEC grade 9 bearings. A thin-walled ( $\left.0.030^{\prime \prime}\right)$ housing supports the PWA and one end of the bearing shaft. An adapter plate supports the other end of the filter wheel shaft, adapts to the lens and blocks unwanted light. The housing and adapter are pinned to one another for precision alignment, and when assembled together they compress the exposed thermal planes on the corners of the PWB by a prescribed amount to provide good thermal transport without crushing the board. The No. 5 stepper motor has a spur gear welded to its shaft, and is located and fixed to the adapter plate. Particle generation is minimized by using a PTFE dichronite coating on the filter wheel teeth, and a low vapor pressure lubricant on the spur gear. During final assembly of this module, the system is hand tuned by moving the stepper motor relative to the filter wheel subassembly to optimally align the meshed gears.

During development, we found that this filter wheel system required an axial load on the bearings. This loading provided frictional damping which allowed the driven wheel to settle within target time limits. Without the damping, the wheel
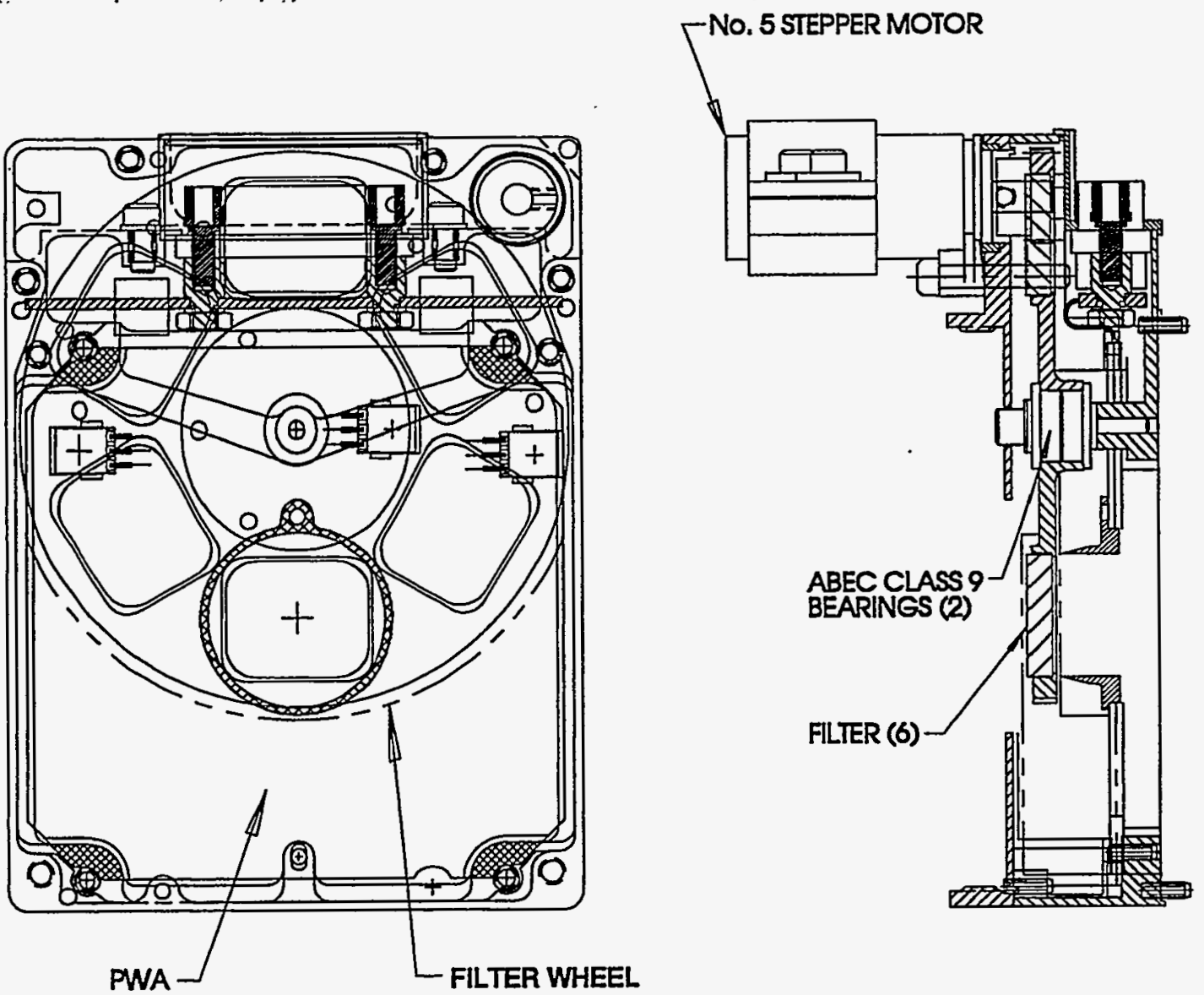

Figure 5. Filter wheel module construction for the UV/Visble camera. 
essentially acted as an undamped system: the wheel would overshoot the intended position resulting in either a missed filter position or "landing" between filters thereby obscuring the field of view. Application of the axial loading, through a set of wavy washers resolved the problem. A series of accelerated life tests, with $>10^{6}$ total steps using simulated mission profiles, were executed to validate the implemented design.

\section{ENGINEERING HOUSEKEEPING DATA CHANNELS}

Several parameters were monitored to track the health of the cameras over the mission duration. These were image quality (dark level), CCD temperature, lens temperature, camera current levels, camera voltage levels and lens heater current. Additionally, switches were monitored to indicate ON/OFF status.

Temperatures were measured with Fenwall LTN-11 thermistors (purchased with calibration curves), with each thermistor bonded in place with Tra-Bond 2151 thermally conductive adhesive. The CCD thermistor was mounted flush with the backside of the alumina carrier package of the CCD, and was captured by a machined slot in the camera housing. The lens thermistor was bonded to a flat on the lens heater clamp, and was left exposed because the camera was wrapped in its own MLI blanket which mitigated radiative heat transfer effects on its measurement.

\section{INTERFACES}

Camera interfaces (optical, mechanical, thermal, electrical and communication) were defined with the spacecraft integrator (Naval Research Laboratory) prior to, and modified during, camera development. An interface control document (ICD), Ref [7], provided working constraints between LLNL and NRL.

The camera is bolted to the spacecraft at the camera mounting plate with three No 4-40 fasteners. Two Al 1100-0 thermal straps were attached at the camera (CCD and stepper motor) and directly to the camera heat pipe (part of the spacecraft Thermal Control System). The camera was thermally isolated by the MLI blanket previously mentioned, and the protective panel used in front of all the cameras. The camera communicated to the spacecraft processor via a synchronous addressable serial interface (SASI) bus protocol based on the Goddard Flight Center (GSFC) 650C custom PMOS process digital integrated circuit. Digital lines were CMOS tri-stated differential line drivers and receivers based on RS-422.

\section{FLIGHT QUALIFICATION DESIGN, ANALYSIS \&TESTING}

Cameras were designed, analyzed, developed and subjected to critical peer review (design reviews and test data reviews). Each camera was subjected to extensive testing to measure compliance with interface definitions and show basic functionality, determine compliance with environmental test requiremnents, and to characterize the electro-optical preformance in response to expected viewing scenes. Prototype units were built to act as a pathfinder during each phase of development testing. These prototypes were also aggressively used in integration activities to find problems early thereby maintaining schedule.

Environmental testing was performed in compliance with the Clementine program guidelines and MIL-STD 1540B "Test Requirements for Space Vehicles". Tests included radiation (for the CCD and electronic components), random vibration, thermal cycling, thermal vacuum and electronic burn-in. Table 2 summarizes the test environments.

\section{CHARACTERIZATION AND CALIBRATION}

Extensive pre-flight calibration data were acquired using an automated calibration facility at LLNL. In a typical calibration configuration, a sensor was mounted inside a dry nitrogen environmental chamber whose temperature was varied systematically over the range -20 to $20^{\circ} \mathrm{C}$, the expected operating temperatures for the mission. Depending on the measurement types the sensors saw either a flat diffused light source or a collimator with various pinholes as the point source. A custom board controlled the sensor parameters from the host computers; the video signal was acquired using a commercial image processor. During data acquisition many thermal parameters such as CCD and chamber temperatures were monitored and recorded as part of the image structure. All calibration processes were highly automated, enabling us to acquire data quickly and reduce operator errors. Pre-flight calibration attempted to cover similar light levels expected from space and the lunar surface and spanned the same camera settings required for lunar mapping phase. 
Table 2. Clementine UV/Visible camera environmental analysis and testing.

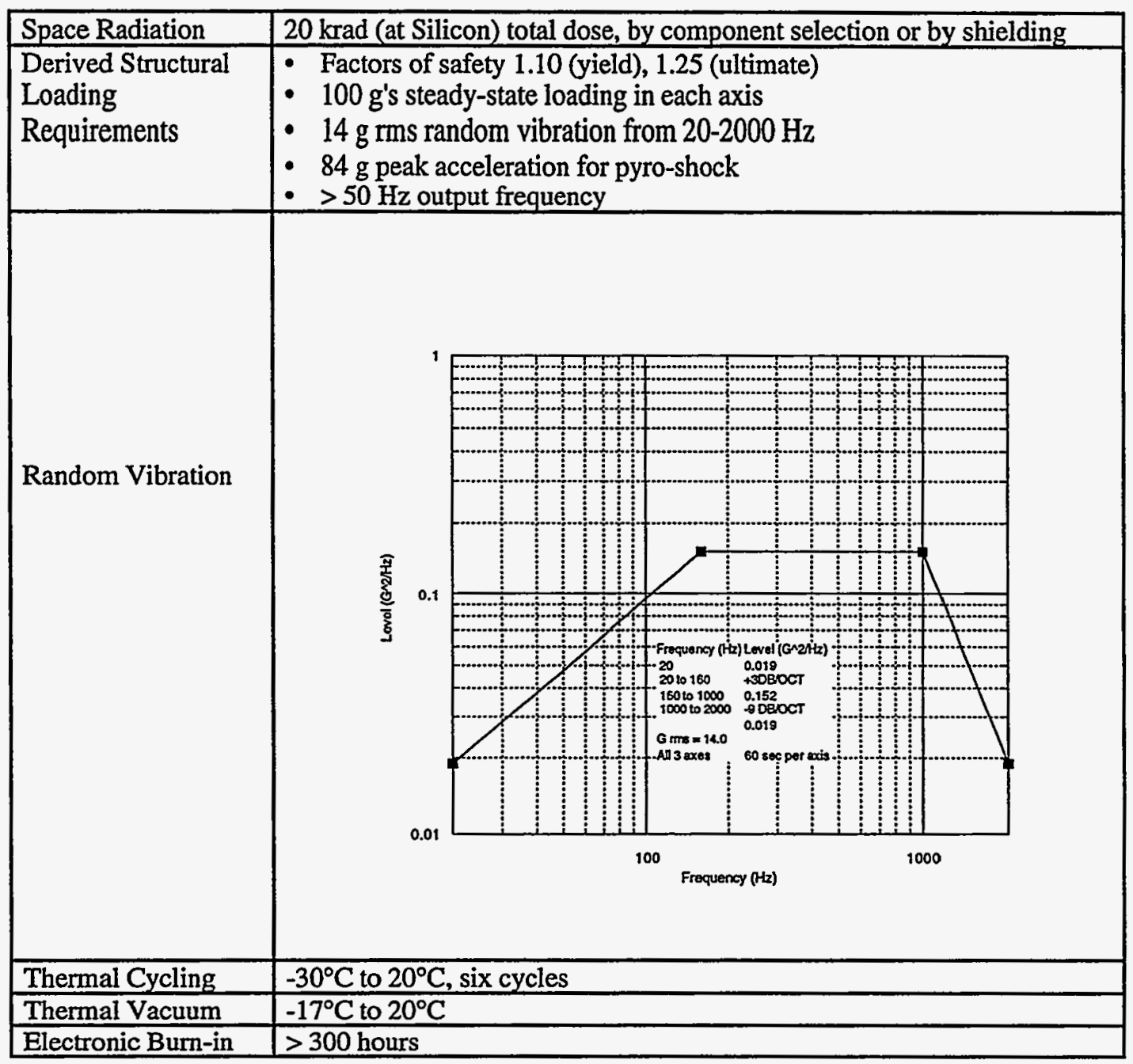

The pre-flight calibration measurements included radiometric sensitivity; CCD uniformity; gain and offset scale factors; temporal/spatial noise; dark noise dependence on CCD temperatures, integration times or input voltage levels, spectral response of $\mathrm{CCD}$; optical distortion map; point spread function and electronic warm-up time. Ref [8] provides a more complete description of these calibrations.

Many pre-flight calibration coefficients were applied to lunar data showing reasonable agreement with expected performance. In-flight calibration data will allow minor corrections for vacuum flight condition and sensor degradation over mission lifetime to be added to the pre-flight calibration results. The final calibration is expected to be better than 5\%.

\section{CLEMENTINE MISSION DATA}

The UV/Visible camera operated throughout the entire Clementine mission and showed no obvious degradation. In the prelunar mapping portion of the mission (LEO/cruise to the moon), the sensitivity of the UV/Visible was verified by imaging whole-earth, whole-moon, and bright stars. Star magnitudes dimmer than magnitude 7.5 were detectable, as predicted by systems analysis calculations. In addition, a series of uncompressed space-looking frames (away from the sun, moon, and earth) were taken at the end - and usually at the beginning — of each lunar imaging pass for dark image calibration and 
sensor performance checks. The UV/Visible consistently showed minimal noise levels in these calibration frames and less than 2 counts dark field increase over the duration of the imaging pass. Dark field signal is consistent with the thermally induced dark signal increase measured in the laboratory.

Lunar mapping operation covered the entire south pole to north pole latitudes in an orbit that deviated $\pm 30^{\circ}$ from sun synchronous over the 60 days of mapping. The orbital plane was aligned so that, approximately half way trough the mission, the Sun, Moon, and orbit were coplanar. Pole-to-pole coverage used roughly 1.5 hours of each 5-hour orbit. Images for lunar mapping were taken often enough to provide roughly $10 \%$ overlap between adjacent frames at the equator. Near the poles, where range to the moon was on the order of $1000 \mathrm{~km}$, the time between the sets of 6-filter position spectral data cubes were on the order of 20 minutes; near the equator (periselene), the frequency increased to roughly 4 minutes between spectral data cubes. Data were generally compressed prior to telemetry. Typical compression ratios were about 11:1, varying slightly with scene content. The compression was adjusted to give less than $1 / 4$ count rms difference between an uncompressed (original) download file and a compressed/decompressed image, as evaluated on selected lunar scenes at the beginning of the orbit.

Camera integration time, offset, and gain controls were set by a spacecraft control file, which switched instructions every $10^{\circ}$ latitude. Near the equator, the UV/Visible camera was operated at the shortest integration time at the lowest gain setting to avoid $A / D$ well saturation. Near the poles, where lunar radiance dropped off severely, the integration time was increased up to a maximum value of $40 \mathrm{msec}$, limited by ground smearing effects at the longer integration times, and the medium gain was used. Signal to noise $(\mathrm{S} / \mathrm{N})$ was limited by digitization noise for all daytime lunar imaging scenes, with the exception of the shortest and longest wavebands in the most extreme polar images, where minor increases over the digitization noise are incurred.

The Clementine satellite returned approximately 1.7 million images, and the Clementine Science Team still is analyzing the data. Preliminary analysis of the UV/Vis images has provided new insights into our view of the Moon (see Fig. 6) and new, mineralogical surface maps of the Moon. It has also shown that in bright extended scenes, electronic and optical artifacts such as veiling glare can add a measurable pedastal to the mean apparent signal from the camera. Additionally, if scenes are sufficiently bright, the integration time must be reduced to a duration that is comparable to the time required to sweep away residual charge. The Thomson CCD uses frame transfer electronic shuttering, which enables photocharge to accumulate even during sweep-out. Consequently, very bright scenes acquire an integrated signal above and beyond that which is intended, and pixel cross-talk increases. For high optical fluxes on it, the CCD never quite empties, and the maximum allowable signal from the detector actually can decrease. As a rule, the latter effects did not appear to contaminate the images away from the lunar equator or over dark Mare regions. Work is in progress at LLNL to reduce the symptoms of the camera artifacts by using a combination of ground-based data and in-flight calibration techniques.

\section{LESSONS LEARNED}

The UV/Visible camera performed well and consistently through the life of the Clementine mission. Cautions to future camera users would be limited to a concern about the maximum brightness that can be detected on the FPA, and the extent of the veiling glare interpretation required for images. Better handling of excess charge (anti-blooming) and better charge flushing before integration would improve the performance and dynamic range of the CCD.

The maximum scene brightness was limited to a value less than the average well capacity of the CCD pixels for short integration times, due to the efficiency of sweeping charge out of the pixel wells prior to integration. The Clementine camera version had a special modification of the readout electronics to repeatedly sweep the charge out of the wells 8 consecutive times prior to the start of integration to increase the allowable imaged scene radiance. Even with this improvement to modify the camera performance complete saturation of the image would have occurred if the bandpass filters were not chosen appropriately.

The veiling glare interpretation will be the most difficult feature of data extraction for the UV/Visible camera. Out of field scattering values require a global mapping model, that must be iteratively solved to yield actual scene radiance values good to $1 \%$. In retrospect, a design of a re-imaging system with a field stop and a LYOT stop, that would effectively eliminate the bulk of the veiling glare, would be a better global design choice for the entire data collection and archival effort. 


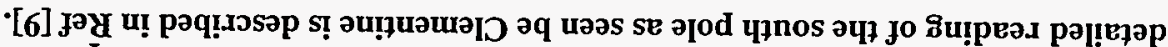

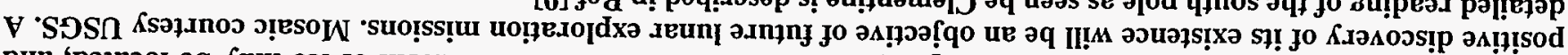

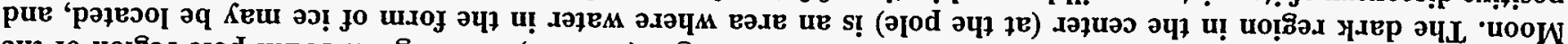

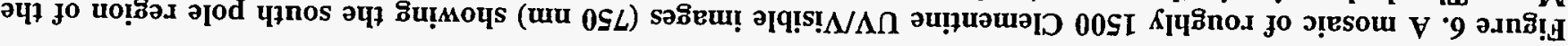

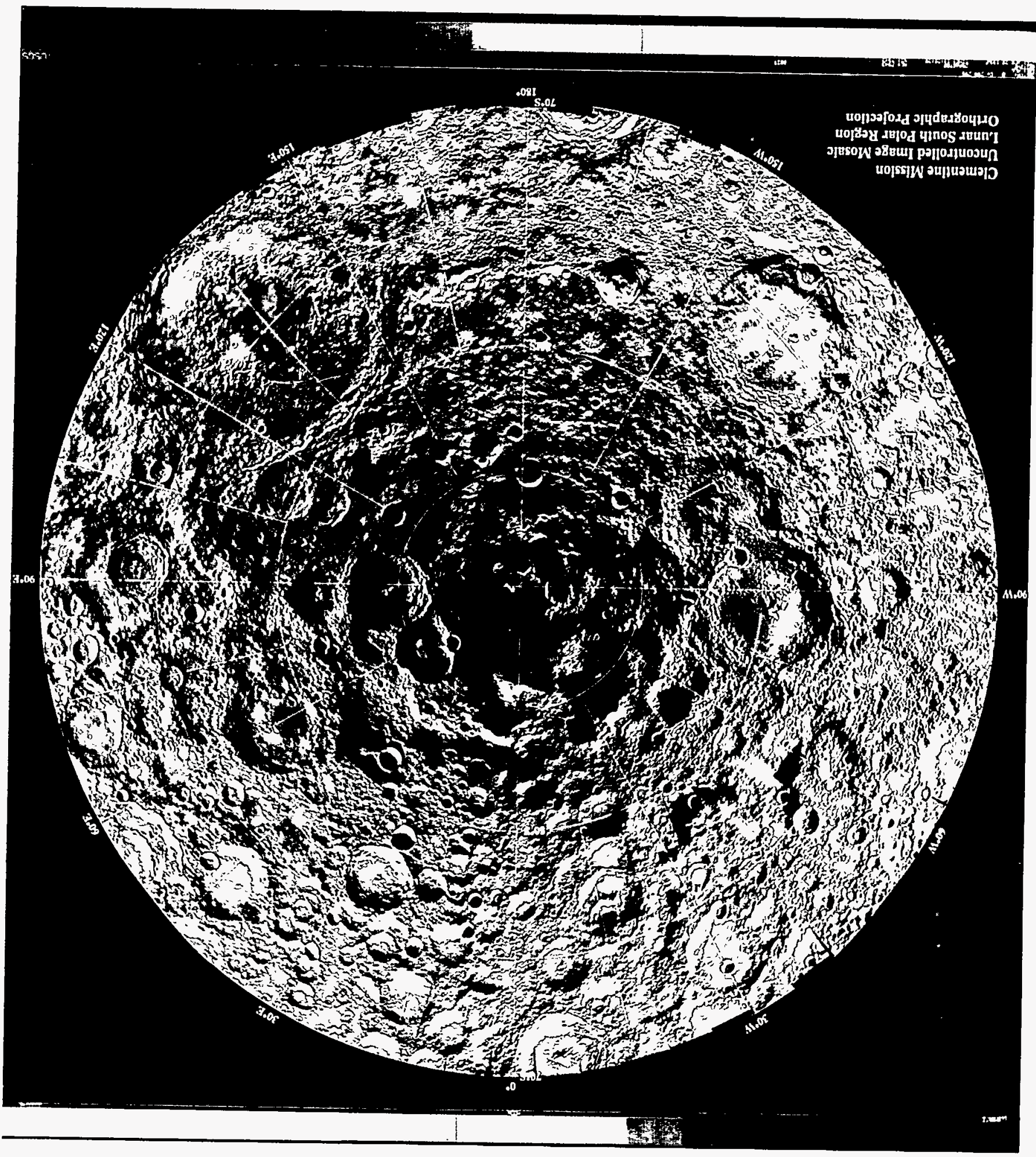




\section{CONCLUSIONS}

The UV/Visible camera performed well and consistently through the life of the Clementine mission, generating a high-quality mineral typing data set for a comprehensive lunar mapping archive It has proven quite space-worthy. All electronic and moving parts (e.g., the filter wheel) functioned as designed whenever the camera was used. Solarization of the optics and cosmic-ray induced defects in the CCD and camera electronics posed no obvious problems. Cautions to future camera users would be limited to a concern about the maximum brightness that can be detected on the FPA and to the extent of the veiling glare interpretation required for images of extended scenes (Note that the extent to which glare-inducing optical contamination may occur varies strongly from one space mission to another and is as much a function of the environment of the camera as of anything). For the Clementine mission, the camera artifacts appear to be generally insignificant. In general, the UV/Vis camera module provides a lightweight, low-power unit that can serve as the basis for other modular spacequalified designs simply by swapping out the optics.

\section{ACKNOWLEDGEMENTS}

This work was performed under the auspices of the U.S. Department of Energy by Lawrence Livermore National Laboratory under contract No. W-7405-ENG-48. Funding was provided by the U.S. Ballistic Missile Defense Office.The lens was designed and built by Optical Corporation of America - Applied Optics under LLNL subcontract B208975.

\section{REFERENCES}

1. J. F. Kordas, et al, "Star Tracker Camera for the Clementine Mission", SPIE paper 2466-10, April 1995.

2. R. E. Priest, et al, "Near-Infrared Camera for the Clementine Mission", SPIE paper 2475-52, April 1995.

3. R. E. Priest, et al, "The Clementine Longwave Infrared Camera", SPIE paper 2475-53, April 1995.

4. A. G. Ledebuhr, et al, "High Resolution Camera and LIDAR Ranging System for the Clementine Mission", SPIE paper 2472-08, April 1995.

5. SCIENCE, Vol 266, S. Nozette, et al, "The Clementine Mission to the Moon: Scientific Overview", plus six additional related articles by various authors, pp 1835 - 1862, 16 Dec 1994.

6. M. J. Shannon, "The Clementine Satellite", LLNL document UCRL-52000-94-6, Energy \& Technology Review, June 1994.

7. R. E. Priest, et al, "Sensor Integration Project UV/Visible Camera Interface Control Document", LLNL document C1-S2-008.

8. H. S. Park, et al, "Caibration of Clementine Sensors", SPIE paper 2474-24, April 1995.

9. E. M. Shoemaker, M. S. Robinson and E. M. Eliason, "The South Pole Region of the Moon as Viewed by Clementine", SCIENCE, Vol 266, pp 1851-1854, 16 Dec 1994.

10. A more complete documentation of the Clementine UV/Visible camera is being published as a formal LLNL UCRL document. Availability is expected in summer 1995. 